\title{
Boundedness for a Class of Generalized Commutators of Fractional Hardy Operators with a Rough Kernel
}

\author{
Xiao $\mathrm{Yu}^{1}$ and Shanzhen $\mathrm{Lu}^{2}$ \\ ${ }^{1}$ Department of Mathematics, Shangrao Normal University, Shangrao 334001, China \\ ${ }^{2}$ School of Mathematical Science, Beijing Normal University, Beijing 100875, China
}

Correspondence should be addressed to Xiao Yu; xyuzju@163.com

Received 7 July 2013; Accepted 1 October 2013

Academic Editor: Csaba Varga

Copyright (C) 2013 X. Yu and S. Lu. This is an open access article distributed under the Creative Commons Attribution License, which permits unrestricted use, distribution, and reproduction in any medium, provided the original work is properly cited.

The authors consider the generalized commutator of fractional Hardy operator with a rough kernel as follows: $\mathscr{H}_{\Omega, A, \beta}^{m} f(x)=$ $1 /\left(|x|^{n-\beta}\right) \int_{|y|<|x|}\left(\Omega(x-y) /|x-y|^{m-1}\right) R_{m}(A ; x, y) f(y) d y$, where $\Omega \in L^{r}\left(\mathbb{S}^{n-1}\right), 0 \leq \beta<n$, and $R_{m}(A ; x, y)=A(x)-$ $\sum_{|\gamma|<m}(1 / \gamma !) D^{\gamma} A(y)(x-y)^{\gamma}$ with $m \in Z^{+}$. The authors prove that $\mathscr{H}_{\Omega, A, \beta}^{m}$ is bounded on Herz type space and $\lambda$-Central Morrey space with $m \geq 1$, which is an open problem for $m>2$.

\section{Introduction}

It is well known that the $\mathrm{C}$ - $\mathrm{Z}$ singular integrals and their commutators have been studied a lot by many mathematicians; please see [1] or [2] for more details. For the generalizations of the commutators of singular integrals, Cohen [3] studied the following generalized commutator $T_{A}^{2}$ which is defined by

$$
\begin{array}{r}
T_{A}^{2} f(x)=\int_{\mathbb{R}^{n}} \frac{\Omega(x-y)}{|x-y|^{n+1}}(A(x)-A(y) \\
-\nabla A(y)(x-y)) f(y) d y
\end{array}
$$

where $\Omega \in L^{1}\left(\mathbb{S}^{n-1}\right)$ is homogeneous of degree zero and satisfies the moment condition

$$
\int_{\mathbb{S}^{n-1}} \Omega(x) x^{\gamma} d \sigma(x)=0
$$

with $|\gamma|=1$. Cohen [3] proved that if $\Omega \in \operatorname{Lip}_{1}\left(\mathbb{S}^{n-1}\right)$ and $\nabla A \in \mathrm{BMO}$, then $T_{A}^{2}$ is bounded on $L^{p}\left(\mathbb{R}^{n}\right)$ with $1<p<$ $\infty$. Later, Cohen and Gosselin [4] considered another type of generalized commutator as follows:

$$
T_{A}^{m} f(x)=\int_{\mathbb{R}^{n}} \frac{\Omega(x-y)}{|x-y|^{n+m-1}} R_{m}(A ; x, y) f(y) d y,
$$

where $R_{m}(A ; x, y)\left(m \in Z^{+}\right)$is defined by $R_{m}(A ; x, y)=$ $A(x)-\sum_{|\gamma|<m}(1 / \gamma !) D^{\gamma} A(y)(x-y)^{\gamma}$, the $m$ th remainder of Taylor series of the function $A$ at $y$ about $x$, and $\Omega$ satisfies the following moment condition:

$$
\int_{\mathbb{S}^{n-1}} \Omega(x) x^{\gamma} d \sigma(x)=0
$$

with $|\gamma|=m-1$. Obviously, if we choose $m=1, T_{A}^{m}$ becomes $[A, T]$, the commutator of $T$ generalized by $A$ and $T$. Furthermore, $T_{A}^{m}$ becomes $T_{A}^{2}$ if we choose $m=2$.

Cohen and Gosselin proved that if $m \geq 2, \Omega \in \operatorname{Lip}_{1}\left(\mathbb{S}^{n-1}\right)$, and the function $A$ has derivatives of order $m-1$ in $\operatorname{BMO}\left(\mathbb{R}^{n}\right)$, then the operator $T_{A}^{m}$ is bounded on $L^{p}\left(\mathbb{R}^{n}\right)$ for $1<p<\infty$. Later, $T_{A}^{m}$ was studied by many mathematicians; please see $[5,6]$ or [7] for more details. Recently, Wang and Zhang [8] gave a new proof of Wu's theorem in [9] by using the $W^{1, p}$ estimate for the elliptic equation of divergence form with partially BMO coefficients and the $L^{p}$ boundedness of the Cohen-Gosselin type generalized commutators proved by Yan in [6]. Furthermore, the method used in [8] is much simpler than that in [9]. Recently, Yu and Tao [7] proved that $T_{A}^{m}$ is bounded on $\lambda$-Central Morrey space. 
Let $f$ be a nonnegative integral on $\mathbb{R}^{+}$: then the Hardy operator is defined by

$$
H f(x)=\frac{1}{x} \int_{0}^{x} f(t) d t, \quad x \neq 0
$$

In 1920, Hardy [10] proved the following inequality:

$$
\|H f\|_{L^{p}\left(\mathbb{R}^{+}\right)} \leq \frac{p}{p-1}\|f\|_{L^{p}\left(\mathbb{R}^{+}\right)},
$$

where $1<p<\infty$ and the constant $p /(p-1)$ is the best possible.

In 2007, Fu et al. [11] introduced the $n$-dimensional fractional type Hardy operator $\mathscr{H}_{\beta}$ as follows:

$$
\mathscr{H}_{\beta} f(x)=\frac{1}{|x|^{n-\beta}} \int_{|t|<|x|} f(t) d t, \quad x \in \mathbb{R}^{n} \backslash\{0\},
$$

where $-n<\beta<n$ and $f$ is a locally integrable function on $\mathbb{R}^{n}$.

Obviously, when $\beta=0, \mathscr{H}_{0}$ is just the $n$-dimensional Hardy operator $\mathscr{H}$ which was proposed by Christ and Grafakos in [12].

In [11], the authors gave the characterization of the $\mathrm{CBMO}^{q}\left(\mathbb{R}^{n}\right)$ by the boundedness of the commutator of the fractional type Hardy operator $\left[\mathscr{H}_{\beta}, b\right]$ on Herz type spaces. Here the $\mathrm{CBMO}^{q}\left(\mathbb{R}^{n}\right)$ space is defined by the following.

Definition 1 (see [13]). Let $1 \leq q<\infty$. A function $f \in$ $L_{\text {loc }}^{q}\left(\mathbb{R}^{n}\right)$ is said to belong to the homogeneous Central BMO space $\mathrm{CBMO}^{q}\left(\mathbb{R}^{n}\right)$ if

\section{$\|b\|_{\mathrm{CB்MO}^{q}\left(\mathbb{R}^{n}\right)}$}

$$
:=\sup _{r>0}\left(\frac{1}{|B(0, r)|} \int_{B(0, r)}\left|f(x)-f_{B}\right|^{q} d x\right)^{1 / q}<\infty,
$$

where $f_{B}=(1 /|B(0, r)|) \int_{B(0, r)} f(x) d x$.

From [14], we know that $\operatorname{BMO}\left(\mathbb{R}^{n}\right) \subset \operatorname{CBMO}^{q}\left(\mathbb{R}^{n}\right)$ for $1 \leq q<\infty$.

The $\mathrm{CBMO}^{q}\left(\mathbb{R}^{n}\right)$ space can be regarded as the space of bounded mean oscillation, a local version of $\mathrm{BMO}\left(\mathbb{R}^{n}\right)$ at the origin. But the famous John-Nirenberg inequality no longer holds in $\mathrm{CBMO}^{q}\left(\mathbb{R}^{n}\right)$.

Now we are interested in the following generalized commutator of Hardy operator:

$$
\mathscr{H}_{A}^{m} f(x)=\frac{1}{|x|^{n}} \int_{|y|<|x|} \frac{1}{|x-y|^{m-1}} f(y) R_{m}(A ; x, y) d y,
$$

where $R_{m}(A ; x, y)=A(x)-\sum_{|\gamma|<m}(1 / \gamma !) D^{\gamma} A(y)(x-y)^{\gamma}$ and $m \in Z^{+}$

In 2010, Lu and Zhao [15] proved that when $m=2, \mathscr{H}_{A}^{2}$ is bounded on Herz type space and Morrey-Herz type space. Later, Gao and $\mathrm{Yu}$ [16] proved that $\mathscr{H}_{A}^{2}$ is bounded on $\lambda$ Central Morrey spaces. However, we would like to point out that the method used in $[15,16]$ cannot apply to the case when $m>2$. An interesting question is whether the boundedness of $\mathscr{H}_{A}^{m}$ on Herz type space or $\lambda$-Central Morrey space still holds with $m>2$. In this paper, we will use a different method to answer this question. Furthermore, we will consider the generalized commutator of fractional Hardy operator with a rough kernel as follows:

$$
\begin{aligned}
& \mathscr{H}_{\Omega, A, \beta}^{m} f(x) \\
& \quad=\frac{1}{|x|^{n-\beta}} \int_{|y|<|x|} \frac{\Omega(x-y)}{|x-y|^{m-1}} f(y) R_{m}(A ; x, y) d y,
\end{aligned}
$$

where $m \in Z^{+}, 0 \leq \beta<n$, and $\Omega \in L^{r}\left(\mathbb{S}^{n-1}\right)$.

In [17], we prove that $\mathscr{H}_{\Omega, A, \beta}^{m}$ is bounded from $L^{p}$ to $L^{q}$ with $1 / p-1 / q=\beta / n$. Furthermore, we study the endpoint estimates of $\mathscr{H}_{\Omega, A, \beta}^{m}$ on $H^{1}$ spaces with $\Omega \in \operatorname{Lip}_{1}\left(\mathbb{S}^{n-1}\right)$ in [17]. In this paper, we will prove that $\mathscr{H}_{\Omega, A, \beta}^{m}$ is bounded on Herz type space and $\lambda$-Central Morrey space when $\Omega \in L^{r}\left(\mathbb{S}^{n-1}\right)$.

\section{Boundedness of $\mathscr{H}_{\Omega, A, \beta}^{m}$ on Herz Type Spaces}

In this section, we will give the boundedness of $\mathscr{H}_{\Omega, A, \beta}^{m}$ on Herz type spaces. First we introduce some notations that will be used throughout this paper.

Let $B_{k}=\left\{x \in \mathbb{R}^{n}:|x| \leq 2^{k}\right\}, C_{k}=B_{k} \backslash B_{k-1}$, and $\chi_{k}=\chi_{C_{k}}$ for $k \in Z$ : here $\chi_{C_{k}}$ is the characteristic function of the set $C_{k}$.

Definition 2 (see [18]). Let $\alpha \in R, 0<p, q \leq \infty$. Then the homogeneous Herz type space $\dot{K}_{q}^{\alpha, p}\left(\mathbb{R}^{n}\right)$ is defined by

$$
\dot{K}_{q}^{\alpha, p}\left(\mathbb{R}^{n}\right)=\left\{f \in L_{\text {loc }}^{q}\left(\mathbb{R}^{n} \backslash\{0\}\right):\|f\|_{\dot{K}_{q}^{\alpha, p}\left(\mathbb{R}^{n}\right)}<\infty\right\},
$$

where $\|f\|_{\dot{K}_{q}^{\alpha, p}\left(\mathbb{R}^{n}\right)}$ is defined as

$$
\|f\|_{\dot{K}_{q}^{\alpha, p}\left(\mathbb{R}^{n}\right)}=\left\{\sum_{k=-\infty}^{\infty} 2^{k \alpha p}\left\|f \chi_{k}\right\|_{L^{q}\left(\mathbb{R}^{n}\right)}^{p}\right\}^{1 / p}
$$

with the usual modifications made when $p=\infty$ or $q=\infty$.

Now we show our main results in this section.

Theorem 3. Suppose $m \geq 2, \Omega \in L^{r}\left(\mathbb{S}^{n-1}\right)$ with $1<r<\infty$, and $A$ has derivatives of order $m-1$ in $C \dot{B} M O^{p_{2}}$ with $n<$ $p_{2}<\infty$. Let $0<s \leq p<\infty, 1<q, p_{1}<\infty, 1 / q=1 / p_{1}+$ $1 / p_{2}-\beta / n$ with $0 \leq \beta<n$. If $1 / r^{\prime}-1 / q-\beta / n>0, r>p_{1}^{\prime}$, $\alpha_{2}=\alpha_{1}+n / p_{2}$ and $\alpha_{2}$ satisfies the following condition:

$$
\alpha_{2}+n / q-n / r^{\prime}-1 / r-n / p_{2}+\beta<0
$$

then there exists a constant $C$, such that

$$
\left\|\mathscr{H}_{\Omega, A, \beta}^{m} f\right\|_{\dot{K}_{q}^{\alpha_{1}, s}} \leq C \sum_{|\gamma|=m-1}\left\|D^{\gamma} A\right\|_{C \dot{B} M O^{p_{2}}}\|f\|_{\dot{K}_{p_{1}}^{\alpha_{2}, p}} .
$$

For $m=1, \mathscr{H}_{\Omega, A, \beta}^{1}$ is just the commutator of Hardy operator; that is, $\mathscr{H}_{\Omega, A, \beta}^{1}=\mathscr{H}_{\Omega, \beta}^{A} f(x)=A(x) \mathscr{H}_{\Omega, \beta} f(x)-$ $\mathscr{H}_{\Omega, \beta}(A f)(x)$. We have the following theorem of $\mathscr{H}_{\Omega, \beta}^{A}$ on Herz type space. 
Theorem 4. Suppose $\Omega \in L^{r}\left(\mathbb{S}^{n-1}\right)$ with $1<r<\infty$ and $A \in C \dot{B} M O^{p_{2}}$. Let $0<s \leq p<\infty, 1<q, p_{1}, p_{2}<\infty, 1 / q=$ $1 / p_{1}+1 / p_{2}-\beta / n$ with $0 \leq \beta<n$. If $1 / r^{\prime}-1 / q-\beta / n>0, r>p_{1}^{\prime}$, $\alpha_{2}=\alpha_{1}+n / p_{2}$, and $\alpha_{2}$ satisfies (13), then there exists a constant $C$, such that

$$
\left\|\mathscr{H}_{\Omega, \beta}^{A} f\right\|_{\dot{K}_{q}^{\alpha_{1}, s}} \leq C\|A\|_{C \dot{B} M O^{p_{2}}}\|f\|_{\dot{K}_{p_{1}}^{\alpha_{2}, p}}
$$

Remark 5. Comparing Theorems 3 and 4, we find that the restrictions on $\alpha_{1}$ and $\alpha_{2}$ are more rigid in Theorem 4 than in Theorem 3, which indicates that $\mathscr{H}_{\Omega, A, \beta}^{m}$ with $m \geq 2$ has better properties than the commutators.

In order to prove Theorems 3 and 4 , we need the following lemmas.

Lemma 6 (see [19]). Let $1<p_{1}, p_{2}<\infty, 0 \leq \beta<n$, and $\beta / n=1 / p_{1}-1 / p_{2}$. If $\Omega \in L^{r}\left(\mathbb{S}^{n-1}\right)$ with $r>p_{1}^{\prime}$, then there exists a constant $C$ independent of $f$, such that

$$
\left\|\mathscr{H}_{\Omega, \beta} f\right\|_{L^{p_{2}}} \leq C\|f\|_{L^{p_{1}}}
$$

where $\mathscr{H}_{\Omega, \beta}$ is defined by

$$
\mathscr{H}_{\Omega, \beta} f(x)=\frac{1}{|x|^{n-\beta}} \int_{|y|<|x|} \Omega(x-y) f(y) d y .
$$

By checking [19] carefully, one can draw the conclusion that if one replaces $\mathscr{H}_{\Omega, \beta} f(x)$ by $\mathscr{H}_{|\Omega|, \beta}|f|(x)$, then (16) still holds.

Lemma 7. Let $m \geq 1,1<p_{1}<p_{2}<\infty$ and $0 \leq \beta<n$. If $A$ has derivatives of order $m-1$ in $L^{r}\left(\mathbb{R}^{n}\right)$ with $1 / p_{2}=1 / p_{1}+$ $1 / r-\beta / n$ and $r>p_{1}^{\prime}$, then one has

$$
\left\|\mathscr{H}_{\Omega, A, \beta}^{m} f\right\|_{L^{p_{2}}} \leq C \sum_{|\gamma|=m-1}\left\|D^{\gamma} A\right\|_{L^{r}}\|f\|_{L^{p_{1}}}
$$

where the constant $C$ is independent of $f$ and $A$.

Proof. From [20, p. 241], we have the following estimates:

$$
\begin{aligned}
\frac{R_{m}(A ; x, y)}{|x-y|^{m-1}} & \leq \frac{R_{m-1}(A ; x, y)}{|x-y|^{m-1}}+C \sum_{|\gamma|=m-1}\left|D^{\gamma} A(y)\right| \\
& \leq C \sum_{|\gamma|=m-1}\left(\left(D^{\gamma} A\right)^{*}(x)+\left(D^{\gamma} A\right)^{*}(y)\right),
\end{aligned}
$$

where $m \geq 1$ and $(f)^{*}$ is the Hardy-Littlewood maximal function of $f$.
Thus we obtain

$$
\begin{aligned}
& \left|\mathscr{H}_{\Omega, A, \beta}^{m} f(x)\right| \\
& =\left|\frac{1}{|x|^{n-\beta}} \int_{|y|<|x|} \frac{\Omega(x-y) f(y)}{|x-y|^{m-1}} R_{m}(A ; x, y) d y\right| \\
& \leq C \frac{1}{|x|^{n-\beta}} \int_{|y|<|x|}|f(y)||\Omega(x-y)| \\
& \quad \times \sum_{|\gamma|=m-1}\left(\left(D^{\gamma} A\right)^{*}(x)+\left(D^{\gamma} A\right)^{*}(y)\right) d y \\
& \leq C \sum_{|\gamma|=m-1}\left[\left(D^{\gamma} A\right)^{*}(x) \mathscr{H}_{|\Omega|, \beta}|f|(x)\right. \\
& \left.+\mathscr{H}_{|\Omega|, \beta}\left(\left(D^{\gamma} A\right)^{*}|f|\right)(x)\right] .
\end{aligned}
$$

By the above estimates, we can get

$$
\begin{aligned}
& \left(\int_{\mathbb{R}^{n}}\left|\mathscr{H}_{\Omega, A, \beta}^{m} f(x)\right|^{q} d x\right)^{1 / q} \\
& \leq C \sum_{|\gamma|=m-1}\left(\left(\int_{\mathbb{R}^{n}}\left|\left(D^{\gamma} A\right)^{*}(x) \mathscr{H}_{|\Omega|, \beta}\right| f|(x)|^{q} d x\right)^{1 / q}\right. \\
& \left.\quad+\left(\int_{\mathbb{R}^{n}}\left|\mathscr{H}_{|\Omega|, \beta}\left(\left(D^{\gamma} A\right)^{*}|f|\right)(x)\right|^{q}\right)^{1 / q}\right) \\
& \leq C \sum_{|\gamma|=m-1}(I+I I) .
\end{aligned}
$$

For the term $I$, let $1 / q=1 / r+1 / l=1 / r+1 / p-\beta / n$; then by the Hölder inequality, Lemma 6 , and the boundedness of Hardy-Littlewood maximal function on $L^{p}$ spaces, we obtain

$$
\begin{aligned}
I & \leq\left(\int_{\mathbb{R}^{n}}\left(D^{\gamma} A\right)^{*}(x)^{r} d x\right)^{1 / r}\left(\int_{\mathbb{R}^{n}}\left|\mathscr{H}_{|\Omega|, \beta}\right| f|(x)|^{l} d x\right)^{1 / l} \\
& \leq C\left\|\left(D^{\gamma} A\right)^{*}\right\|_{L^{r}}\|f\|_{L^{p}} \\
& \leq C\left\|D^{\gamma} A\right\|_{L^{r}}\|f\|_{L^{p}} .
\end{aligned}
$$

For the term $I I$, let $1 / q=1 / t-\beta / n=1 / r+1 / p-\beta / n$; then by the Hölder inequality and Lemma 6 , we have

$$
\begin{aligned}
I I & \leq C\left(\int_{\mathbb{R}^{n}}\left|\left(D^{\gamma} A\right)^{*}(x) f(x)\right|^{t} d x\right)^{1 / t} \\
& \leq C\left\|\left(D^{\gamma} A\right)^{*}\right\|_{L^{r}}\|f\|_{L^{p}} \\
& \leq C\left\|D^{\gamma} A\right\|_{L^{r}}\|f\|_{L^{p}} .
\end{aligned}
$$

Combining the estimates of $I$ and $I I$, we finish the proof of Lemma 7. 
Lemma 8 (see [4]). Let $b$ be a function on $\mathbb{R}^{n}$ with $m$ th order derivatives in $L_{\text {loc }}^{q}\left(\mathbb{R}^{n}\right)$ for some $q>n$. Then

$$
\begin{aligned}
\left|R_{m}(b ; x, y)\right| \leq & C_{m, n}|x-y|^{m} \\
& \times \sum_{|\gamma|=m}\left(\frac{1}{|\widetilde{Q}(x, y)|} \int_{\widetilde{Q}(x, y)}\left|D^{\gamma} b(z)\right|^{q} d z\right)^{1 / q},
\end{aligned}
$$

where $\widetilde{Q}(x, y)$ is the cube centered at $x$ having diameter $5 \sqrt{n}|x-y|$.

Lemma 9 (see [5]). Suppose that $f \in C \dot{B} M O^{q}\left(\mathbb{R}^{n}\right), 1 \leq q<$ $\infty$, and $r_{1}, r_{2}>0$; then

$$
\begin{gathered}
\left(\frac{1}{\left|B\left(0, r_{1}\right)\right|} \int_{B\left(0, r_{1}\right)}\left|f(x)-f_{B\left(0, r_{2}\right)}\right|^{q} d x\right)^{1 / q} \\
\leq C\left(1+\left|\log \left(\frac{r_{1}}{r_{2}}\right)\right|\right)\|f\|_{C \dot{B} M O^{q}} .
\end{gathered}
$$

Proof of Theorem 3. To prove Theorem 3, first we split each $f$ as

$$
f(x)=\sum_{i=-\infty}^{+\infty} f(x) \chi_{i}(x)=\sum_{i=-\infty}^{+\infty} f_{i}(x)
$$

then we have

$$
\begin{aligned}
\left\|\mathscr{H}_{\Omega, A, \beta}^{m} f \chi_{k}\right\|_{L^{q}}^{q} & \int_{C_{k}}\left(\int_{B(0,|x|)} \frac{|f(y)||\Omega(x-y)|}{|x-y|^{m-1}} R_{m}(A ; x, y) d y\right)^{q} \\
& \times|x|^{(\beta-n) q} d x \\
\leq & \int_{C_{k}}\left(\sum_{i=-\infty}^{k} \int_{C_{i}} \frac{|\Omega(x-y) f(y)|}{|x-y|^{m-1}} R_{m}(A ; x, y) d y\right)^{q} \\
& \times|x|^{(\beta-n) q} d x \quad \int_{C_{k}}\left(\sum_{i=-\infty}^{k-3} \int_{C_{i}} \frac{|\Omega(x-y) f(y)|}{|x-y|^{m-1}} R_{m}(A ; x, y) d y\right)^{q} \\
& \times|x|^{(\beta-n) q} d x \\
+ & C \int_{C_{k}}\left(\sum_{i=k-2}^{k} \int_{C_{i}} \frac{|\Omega(x-y) f(y)|}{|x-y|^{m-1}} R_{m}(A ; x, y) d y\right)^{q} \\
& \times|x|^{(\beta-n) q} d x \\
= & \left(I_{1}+I_{2}\right) .
\end{aligned}
$$

For the term $I_{1}$, we denote $A_{k}(x)=A(x)-$ $\sum_{|\gamma|=m-1}(1 / \gamma !) m_{B_{k}}\left(D^{\gamma} A\right) x^{\gamma}$; then it is easy to check $R_{m}(A ; x, y)=R_{m}\left(A_{k} ; x, y\right)$. By the fact that $x \in C_{k}$, $y \in C_{i}$ with $i \leq k-3$, we have $|x-y| \sim|x| \sim 2^{k}$. As $p_{2}>n$, then by Lemmas 8 and 9 , we obtain

$$
\begin{aligned}
& \left|R_{m}\left(A_{k} ; x, y\right)\right| \\
& \leq\left|R_{m-1}\left(A_{k} ; x, y\right)\right|+\sum_{|\gamma|=m-1} \frac{1}{\gamma !}\left|D^{\gamma} A_{k}(x)\right||x-y|^{m-1} \\
& \leq C|x-y|^{m-1} \sum_{|\gamma|=m-1}\left\{\left(\frac{1}{|\widetilde{Q}(x, y)|}\right.\right. \\
& \left.\times \int_{\widetilde{Q}(x, y)}\left|D^{\gamma} A_{k}(z)\right|^{p_{2}} d z\right)^{1 / p_{2}} \\
& \left.+\left|D^{\gamma} A_{k}(y)\right|\right\} \\
& \leq C|x-y|^{m-1} \sum_{|\gamma|=m-1}\left(\left\|D^{\gamma} A\right\|_{\mathrm{CB} M O^{p_{2}}}+\left|D^{\gamma} A_{k}(y)\right|\right) .
\end{aligned}
$$

As $|x-y| \sim|x| \sim 2^{k}$ and $1-1 / p_{1}-1 / p_{2}-1 / r=1 / r^{\prime}-1 / q-$ $\beta / n>0$, then by the Hölder inequality, we have

$$
\begin{aligned}
& I_{1} \leq C \int_{C_{k}}\left(\sum_{i=-\infty}^{k-3} \int_{C_{i}}|\Omega(x-y) f(y)|\right. \\
& \left.\times \sum_{|\gamma|=m-1}\left(\left\|D^{\gamma} A\right\|_{\mathrm{CBMO}^{p_{2}}}+\left|D^{\gamma} A_{k}(y)\right|\right) d y\right)^{q} \\
& \times|x|^{(\beta-n) q} d x \\
& \leq C 2^{-k(n-\beta) q} \int_{C_{k}}\left(\sum_{i=-\infty}^{k-3} \int_{C_{i}}|f(y)||\Omega(x-y)|\right. \\
& \times \sum_{|\gamma|=m-1}\left(\left\|D^{\gamma} A\right\|_{\mathrm{CB}^{\prime M O} O^{p_{2}}}\right. \\
& \left.\left.+\left|D^{\gamma} A_{k}(y)\right|\right) d y\right)^{q} d x \\
& \leq C 2^{-k(n-\beta) q} \int_{C_{k}} \sum_{i=-\infty}^{k-3}\left(\int_{C_{i}}|f(y)|^{p_{1}} d y\right)^{q / p_{1}} \\
& \times\left(\int_{C_{i}}|\Omega(x-y)|^{r} d y\right)^{q / r} \\
& \times\left(\sum_{|\gamma|=m-1} \int_{C_{i}}\left(\left\|D^{\gamma} A\right\|_{\mathrm{CBMMO}^{p_{2}}}+\left|D^{\gamma} A_{k}(y)\right|\right)^{p_{2}} d y\right)^{q / p_{2}} \\
& \times\left|C_{i}\right|^{q\left(1-1 / p_{1}-1 / p_{2}-1 / r\right)} d x .
\end{aligned}
$$


As

$$
\begin{aligned}
& \left(\int_{C_{i}}\left(\left\|D^{\gamma} A\right\|_{\mathrm{CBMMO}^{p_{2}}}+\left|D^{\gamma} A_{k}(y)\right|\right)^{p_{2}} d y\right)^{1 / p_{2}} \\
& \leq\left(\int _ { C _ { i } } \left(\left\|D^{\gamma} A\right\|_{\mathrm{CBMOO}^{p_{2}}}\right.\right. \\
& \left.\left.\quad+\left|D^{\gamma} A_{k}(y)-m_{B_{k}}\left(D^{\gamma} A\right)(y)\right|\right)^{p_{2}} d y\right)^{1 / p_{2}} \\
& \leq C\left\|D^{\gamma} A\right\|_{\mathrm{CB}^{\prime} M O^{p_{2}}}\left|C_{i}\right||k-i|, \\
& \int_{C_{i}}|\Omega(x-y)|^{r} d y \\
& \leq \int_{|x|-2^{i}}^{|x|+2^{i}} \int_{\mathbb{S}^{n-1}}\left|\Omega\left(y^{\prime}\right)\right|^{r} d \sigma\left(y^{\prime}\right) r^{n-1} d r \leq C 2^{k n-k+i},
\end{aligned}
$$

we obtain the following estimates:

$$
\begin{aligned}
I_{1} \leq C \sum_{|\gamma|=m-1} & \left\|D^{\gamma} A\right\|_{\mathrm{CB்MO}^{p_{2}}}^{q}|k-i|^{q} 2^{-k n q+k \beta q} \\
& \times 2^{i n\left(1-1 / p_{1}-1 / p_{2}-1 / r\right) q} \\
& \times 2^{((k(n-1)+i) / r) q+\left(i n / p_{2}\right) q} \\
& \times \int_{C_{k}} \sum_{i=-\infty}^{k-3}\left\|f_{i}\right\|_{L^{p_{1}}}^{q} d x \\
\leq C \sum_{i=-\infty}^{k-3}( & |k-i| 2^{i n\left(1-1 / p_{1}-1 / p_{2}-1 / r\right)} \\
& \times 2^{-k n+k \beta+(k(n-1)+i) / r+i n / p_{2}+k n / q} \\
& \left.\times\left\|f_{i}\right\|_{L^{p_{1}}} \sum_{|\gamma|=m-1}\left\|D^{\gamma} A\right\|_{\mathrm{CBMMO} \mathrm{p}^{p_{2}}}\right)^{q} .
\end{aligned}
$$

For the term $I_{2}$, we choose $\phi \in C_{0}^{\infty}\left(\mathbb{R}^{n}\right)$ satisfying $\operatorname{supp} \phi \subset B(0,4)$ as well as $\phi \equiv 1$ in $B(0,2)$ and we set $L=\max \left\{\left\|D^{\gamma} \phi\right\|_{L^{\infty}},|\gamma| \leq m-1\right\}$. Let $y_{0} \in B_{k+4}$ and $A_{k}^{\phi}(x)=R_{m-1}\left(A_{k} ; x, y_{0}\right) \phi\left(2^{-k} x\right)$. Then it is easy to see that $R_{m}(A ; x, y)=R_{m}\left(A_{k}^{\phi} ; x, y\right)=R_{m}\left(A_{k} ; x, y\right)$ for $x \in B_{k}$ and $y \in B_{i}$ with $k-2 \leq i \leq k$. Thus we get

$$
\mathscr{H}_{\Omega, A, \beta}^{m} f_{i}(x)=\mathscr{H}_{\Omega, A_{k}^{\phi}, \beta}^{m} f_{i}(x)=\mathscr{H}_{\Omega, A_{k}, \beta}^{m} f_{i}(x) .
$$

Thus by Lemma 7, we have

$$
\begin{aligned}
I_{2} \leq C \sum_{i=k-2}^{k} \int_{C_{k}}\left(\frac{1}{|x|^{n-\beta}} \int_{|y|<|x|} \frac{\left|\Omega(x-y) f_{i}(y)\right|}{|x-y|^{m-1}}\right. \\
\left.\quad \times R_{m}\left(A_{k}^{\phi} ; x, y\right) d y\right)^{q} d x \\
\leq C \sum_{i=k-2}^{k}\left\|\mathscr{H}_{\Omega, A_{k}^{m}, \beta}^{m} f_{i}\right\|_{L^{q}}^{q} \\
\leq C \sum_{i=k-2}^{k}\left\|f_{i}\right\|_{L^{p_{1}}}^{q} \sum_{|\gamma|=m-1}\left\|D^{\gamma} A_{k}^{\phi}\right\|_{L^{p_{2}}}^{q} .
\end{aligned}
$$

From [5, p.80], we have the following estimates:

$$
\left\|D^{\gamma} A_{k}^{\phi}\right\|_{L^{p_{2}}} \leq C 2^{k n / p_{2}} \sum_{|\gamma|=m-1}\left\|D^{\gamma} A\right\|_{\mathrm{CB \dot {MO }}^{p_{2}}},
$$

where $C$ is dependent on $L$. Thus we get

$$
I_{2} \leq C \sum_{i=k-2}^{k}\left\|f_{i}\right\|_{L^{p_{1}}}^{q} 2^{k n q / p_{2}} \sum_{|\gamma|=m-1}\left\|D^{\gamma} A\right\|_{\mathrm{CBMMO}^{p_{2}}}^{q} .
$$

As $0<s \leq p<\infty$, we obtain the following estimates:

$$
\begin{aligned}
& \left\{\sum_{k=-\infty}^{+\infty} 2^{k \alpha_{1} s}\left\|\mathscr{H}_{\Omega, A, \beta}^{m} f \chi_{k}\right\|_{L^{q}}^{s}\right\}^{1 / s} \\
& \leq\left\{\sum_{k=-\infty}^{+\infty} 2^{k \alpha_{1} p}\left\|\mathscr{H}_{\Omega, A, \beta}^{m} f \chi_{k}\right\|_{L^{q}}^{p}\right\}^{1 / p} \\
& \leq C \sum_{|\gamma|=m-1}\left\|D^{\gamma} A\right\|_{\mathrm{CBMOO}^{p_{2}}} \\
& \times\left\{\sum_{k=-\infty}^{+\infty} 2^{k \alpha_{1} p}\right. \\
& \times \sum_{i=-\infty}^{k-3}\left(|k-i| 2^{i n\left(1-1 / p_{1}-1 / p_{2}-1 / r\right)}\left\|f_{i}\right\|_{L^{p_{1}}}\right. \\
& \left.\left.\times 2^{\left.-k n+k \beta+(k(n-1)+i) / r+i n / p_{2}\right)+k n / q}\right)^{p}\right\}^{1 / p} \\
& +C \sum_{|\gamma|=m-1}\left\|D^{\gamma} A\right\|_{\mathrm{CB}^{\prime} M O^{p_{2}}} \\
& \times\left\{\sum_{k=-\infty}^{+\infty} 2^{k \alpha_{1} p} \sum_{i=k-2}^{k}\left\|f_{i}\right\|_{L^{p_{1}}}^{p} 2^{\left(k n / p_{2}\right) p}\right\}^{1 / p} \\
& =C(A+B) \text {. }
\end{aligned}
$$


For the term $A$, we have

A

$$
\begin{aligned}
& =\sum_{|\gamma|=m-1}\left\|D^{\gamma} A\right\|_{\mathrm{CBMO}^{p_{2}}} \\
& \times\left\{\sum_{k=-\infty}^{+\infty} 2^{k \alpha_{1} p}\right. \\
& \quad \times \sum_{i=-\infty}^{k-3}\left(|k-i| 2^{i n\left(1-1 / p_{1}-1 / p_{2}-1 / r\right)} 2^{-k n+k \beta}\right. \\
& \left.\left.\quad \times 2^{(k(n-1)+i) / r+i n / p_{2}+k n / q}\left\|f_{i}\right\|_{L^{p_{1}}}\right)^{p}\right\}^{1 / p}
\end{aligned}
$$$$
\leq C \sum_{|\gamma|=m-1}\left\|D^{\gamma} A\right\|_{\mathrm{CBMO}^{p_{2}}}
$$$$
\times\left\{\sum _ { k = - \infty } ^ { + \infty } \sum _ { i = - \infty } ^ { k - 3 } \left(|k-i| 2^{(k-i)\left(n / q-n / r^{\prime}+\beta-1 / r-n / p_{2}\right)}\right.\right.
$$$$
\times 2^{(k-i)\left(\alpha_{1}+n / p_{2}\right)}
$$$$
\left.\left.\times 2^{i\left(\alpha_{1}+n / p_{2}\right)}\left\|f_{i}\right\|_{L^{p_{1}}}\right)^{p}\right\}^{1 / p} .
$$

When $0<p \leq 1$, by condition (13), we get

$$
\begin{aligned}
& A^{p} \leq C \sum_{|\gamma|=m-1}\left\|D^{\gamma} A\right\|_{\mathrm{CB} M O^{p_{2}}}^{p} \\
& \times \sum_{k=-\infty}^{+\infty}\left(\sum_{i=-\infty}^{k-3} 2^{(k-i)\left(n / q-n / r^{\prime}-1 / r-n / p_{2}+\beta\right)}\right. \\
& \times 2^{(k-i)\left(\alpha_{1}+n / p_{2}\right)} \\
& \left.\times|k-i| 2^{i\left(\alpha_{1}+n / p_{2}\right)}\left\|f_{i}\right\|_{L^{p_{1}}}\right)^{p} \\
& \leq C \sum_{|\gamma|=m-1}\left\|D^{\gamma} A\right\|_{\mathrm{CB}^{\prime} \mathrm{MO}^{p_{2}}}^{p} \\
& \times \sum_{k=-\infty}^{+\infty} 2^{i \alpha_{2} p}\left(\sum_{i=-\infty}^{k-3}|k-i|\right. \\
& \times 2^{(k-i)\left(n / q-n / r^{\prime}-1 / r-n / p_{2}+\beta+\alpha_{2}\right)} \\
& \left.\times\left\|f_{i}\right\|_{L^{p_{1}}}\right)^{p} \\
& \leq C \sum_{|\gamma|=m-1}\left\|D^{\gamma} A\right\|_{\mathrm{CB} M O^{p_{2}}}^{p}\|f\|_{\dot{K}_{p_{1}}^{\alpha_{2}, p}}^{p} .
\end{aligned}
$$

When $p \geq 1$, by the Hölder inequality and condition (13), we have

$$
\begin{aligned}
& A^{p} \\
& \leq C \sum_{|\gamma|=m-1}\left\|D^{\gamma} A\right\|_{\mathrm{CBMO}^{p_{2}}}^{p} \\
& \times \sum_{k=-\infty}^{+\infty} 2^{k \alpha_{1} p}\left(\sum_{i=-\infty}^{k-3}|k-i|\right. \\
& \times 2^{(k-i)\left(n / q-n / r^{\prime}-1 / r-n / p_{2}+\beta\right)} \\
& \left.\times 2^{k n / p_{2}}\left\|f_{i}\right\|_{L^{p_{1}}}\right)^{p} \\
& \leq C \sum_{|\gamma|=m-1}\left\|D^{\gamma} A\right\|_{\mathrm{CBMO}^{p_{2}}}^{p} \\
& \times \sum_{k=-\infty}^{+\infty}\left(\sum_{i=-\infty}^{k-3}|k-i| 2^{k \alpha_{1}} 2^{(k-i)\left(n / q-n / r^{\prime}-1 / r-n / p_{2}+\beta\right)}\right. \\
& \left.\times 2^{k n / p_{2}}\left\|f_{i}\right\|_{L^{p_{1}}}\right)^{p} \\
& \leq C \sum_{|\gamma|=m-1}\left\|D^{\gamma} A\right\|_{\mathrm{CBMO}^{p_{2}}}^{p} \\
& \times \sum_{k=-\infty}^{+\infty}\left(\sum_{i=-\infty}^{k-3}|k-i| 2^{i \alpha_{2}} 2^{(k-i)\left(n / q-n / r^{\prime}-1 / r-n / p_{2}+\beta+\alpha_{2}\right)}\right. \\
& \left.\times\left\|f_{i}\right\|_{L^{p_{1}}}\right)^{p} \\
& \leq C \sum_{|\gamma|=m-1}\left\|D^{\gamma} A\right\|_{\mathrm{CBMO}^{p_{2}}}^{p} \\
& \times \sum_{k=-\infty}^{+\infty} \sum_{i=-\infty}^{k-3} 2^{i \alpha_{2} p}\left\|f_{i}\right\|_{L^{p}}^{p} 2^{p(k-i)\left(n / q-n / r^{\prime}-1 / r-n / p_{2}+\beta+\alpha_{2}\right) / p} \\
& \times\left(\sum_{i=-\infty}^{k-3}|k-i|^{p^{\prime}} 2^{\left((k-i) / p^{\prime}\right)\left(n / q-n / r^{\prime}-1 / r-n / p_{2}+\beta+\alpha_{2}\right) p^{\prime}}\right)^{p / p^{\prime}} \\
& \leq C \sum_{|\gamma|=m-1}\left\|D^{\gamma} A\right\|_{\mathrm{CBMO}^{p_{2}}}^{p} \\
& \times \sum_{i=-\infty}^{+\infty} \sum_{k=i+3}^{\infty} 2^{i p\left(\alpha_{1}+n / p_{2}\right)} 2^{(k-i)\left(n / q-n / r^{\prime}-1 / r-n / p_{2}+\beta+\alpha_{2}\right)}\left\|f_{i}\right\|_{L^{p_{1}}} \\
& \times\left(\sum_{i=-\infty}^{k-3}|k-i|^{p^{\prime}} 2^{(k-i)\left(n / q-n / r^{\prime}-1 / r-n / p_{2}+\beta+\alpha_{2}\right)}\right)^{p / p^{\prime}}
\end{aligned}
$$




$$
\begin{aligned}
& \leq C \sum_{|\gamma|=m-1}\left\|D^{\gamma} A\right\|_{\mathrm{CBMO}^{p_{2}}}^{p} \sum_{i=-\infty}^{+\infty} 2^{i \alpha_{2} p}\left\|f_{i}\right\|_{L^{p_{1}}}^{p} \\
& \leq C \sum_{|\gamma|=m-1}\left\|D^{\gamma} A\right\|_{\mathrm{CB}^{\prime} M O^{p_{2}}}^{p}\|f\|_{\dot{K}_{p_{1}}^{\alpha_{2}, p} .}^{p}
\end{aligned}
$$

For the term $B$, we have the following estimates:

$$
\begin{aligned}
B & \leq C \sum_{|\gamma|=m-1}\left\|D^{\gamma} A\right\|_{\mathrm{CB} M O^{p_{2}}}\left\{\sum_{k=-\infty}^{+\infty} 2^{k \alpha_{1} p} \sum_{i=k-2}^{k} 2^{k n p / p_{2}}\left\|f_{i}\right\|_{L^{p_{1}}}^{p}\right\}^{1 / p} \\
& \leq C \sum_{|\gamma|=m-1}\left\|D^{\gamma} A\right\|_{\mathrm{CB்MO}^{p_{2}}}\left(\sum_{i=-\infty}^{+\infty} 2^{i \alpha_{2} p}\left\|f_{i}\right\|_{L^{p_{1}}}^{p}\right)^{1 / p} \\
& \leq C \sum_{|\gamma|=m-1}\left\|D^{\gamma} A\right\|_{\mathrm{C \dot {B } M O}^{p_{2}}}\|f\|_{\dot{K}_{p_{1}}^{\alpha_{2}, p} .}
\end{aligned}
$$

Combining the estimates of $A$ and $B$, we finish the proof of Theorem 3.

Proof of Theorem 4. The proof of Theorem 4 is quite similar and much easier than Theorem 3 and we omit the details here.

\section{Boundedness of $\mathscr{H}_{\Omega, A, \beta}^{m}$ on $\lambda$-Central Morrey Spaces}

In [21], Wiener gave a way to describe the behavior of a function at the infinity. Later, Beurling [22] extended Weiner's idea and introduced a pair of dual Banach spaces, $A^{q}$ and $B^{q^{\prime}}$, with $1 / q+1 / q^{\prime}=1$. In [23], Feichtinger proved that $B^{q}$ can be described as

$$
\|f\|_{B^{q}}=\sup _{k \geq 0}\left(2^{-k n / q}\left\|f \chi_{k}\right\|_{L^{q}}\right)<\infty,
$$

where $\chi_{0}$ is the characterization of the unit ball $\left\{x \in \mathbb{R}^{n}\right.$ : $|x| \leq 1\}$ and $\chi_{k}$ is defined as in Section 2.

Now by duality, the space $A^{q}$, which is called the Beurling algebra, can be described by

$$
\|f\|_{A^{q}}=\sum_{k=0}^{\infty} 2^{k n / q^{\prime}}\left\|f \chi_{k}\right\|_{L^{q}}<\infty .
$$

Later, Chen and Lau [24] as well as García-Cuerva [25] introduced atomic spaces $H A^{q}\left(\mathbb{R}^{n}\right)$ associated with the Buerling algebra $A^{q}$ and the dual space of $H A^{q}\left(\mathbb{R}^{n}\right)$ can be described by

$$
\begin{aligned}
& \|f\|_{\mathrm{CBMO}^{q}} \\
& \quad:=\sup _{R \geq 1}\left(\frac{1}{|B(0, R)|} \int_{B(0, R)}\left|f(x)-f_{B(0, R)}\right|^{q} d x\right)^{1 / q}<\infty ;
\end{aligned}
$$

here the $\mathrm{CBMO}^{q}$ can be regarded as the inhomogeneous central BMO spaces.

In 2000, Alvarez et al. [26] introduced the $\lambda$-Central bounded mean oscillation space and $\lambda$-Central Morrey space, respectively.

Definition 10 (see [26]). Given $\lambda<1 / n, 1<q<\infty$, then a function $f \in L_{\mathrm{loc}}^{q}\left(\mathbb{R}^{n}\right)$ is said to belong to the $\lambda$-Central bounded mean oscillation space $\mathrm{CBMO}^{q, \lambda}\left(\mathbb{R}^{n}\right)$ if

$$
\begin{aligned}
& \|f\|_{\text {Св̈мO } 0^{q, \lambda}} \\
& :=\sup _{R>0}\left(\frac{1}{|B(0, R)|^{1+\lambda q}} \int_{B(0, R)}\left|f(x)-f_{B(0, R)}\right|^{q} d x\right)^{1 / q}<\infty .
\end{aligned}
$$

Definition 11 (see [26]). Let $\lambda \in \mathbb{R}$ and $1<q<\infty$. Then the $\lambda$-Central Morrey space $\dot{E}^{q, \lambda}\left(\mathbb{R}^{n}\right)$ is defined by

$$
\|f\|_{\dot{E}^{q}, \lambda}=\sup _{R>0}\left(\frac{1}{|B(0, R)|^{1+\lambda q}} \int_{B(0, R)}|f(x)|^{q} d x\right)^{1 / q}<\infty .
$$

From [27], we know that if $1<q_{1}<q_{2}<\infty$, we obtain $\dot{E}^{q_{2}, \lambda} \subset \dot{E}^{q_{1}, \lambda}$ for $\lambda \in R$ and $\mathrm{CBMO}^{q_{2}, \lambda} \subset \mathrm{CBMO}^{q_{1}, \lambda}$ for $\lambda<$ $1 / n$. Furthermore, when $\lambda<-1 / q, \dot{E}^{q, \lambda}$ reduces to $\{0\}$ and $\mathrm{CB} M O^{q, \lambda}$ reduces to the space of constant functions. When $\lambda=-1 / q, \mathrm{CBMO}^{q, \lambda}$ coincides with $L^{q}\left(\mathbb{R}^{n}\right)$ modulo constant and $\dot{E}^{q, \lambda}=L^{q}$.

In 2011, Fu et al. [19] proved the boundedness of the commutator of fractional Hardy operator with a rough kernel on $\lambda$-Central Morrey space. Later, Fu et al. [28] proved the boundness of the weighted Hardy operator and its commutator on $\lambda$-Central Morrey space. In this paper, we will give the boundedness of $\mathscr{H}_{\Omega, A, \beta}^{m}$ on $\lambda$-Central Morrey space with $m \geq 1$.

Our results can be stated as follows.

Theorem 12. Suppose $m \geq 2, n<p_{2}<\infty, 1<p_{1}<\infty$, $1 / q=1 / p_{1}+1 / p_{2}-\beta / n$ with $0 \leq \beta<n$ and $\lambda=\lambda_{1}+\lambda_{2}+\beta / n$. Let $\Omega \in L^{r}\left(\mathbb{S}^{n-1}\right)$ with $1 / r^{\prime}>\beta / n+1 / q$, and $A$ has derivatives of order $m-1$ in $C \dot{B} M O^{p_{2}, \lambda_{2}}$. If $r>p_{1}^{\prime}, \lambda_{1}>-1 / p_{1}$, and $q \lambda+1>0$, then one has

$$
\left\|\mathscr{H}_{\Omega, A, \beta}^{m} f\right\|_{\dot{E}^{q, \lambda}} \leq C \sum_{|\gamma|=m-1}\left\|D^{\gamma} A\right\|_{C \dot{B} M O^{p_{2}, \lambda_{2}}}\|f\|_{\dot{E}^{p_{1}, \lambda_{1}}},
$$

where the constant $C$ is independent of $f$ and $A$.

For the case $m=1$, we have the following theorem.

Theorem 13. Suppose $1<p_{1}, p_{2}<\infty, 1 / q=1 / p_{1}+1 / p_{2}-$ $\beta / n$ with $0 \leq \beta<n$ and $\lambda=\lambda_{1}+\lambda_{2}+\beta / n$. Let $\Omega \in L^{r}\left(\mathbb{S}^{n-1}\right)$ with $1 / r^{\prime}>\beta / n+1 / q$ and $A \in C \dot{B} M O^{p_{2}}, \lambda_{2}$. If $\lambda_{1}>-1 / p_{1}, r>$ $p_{1}^{\prime}$, and $q \lambda+1>0$, then one has

$$
\left\|\mathscr{H}_{\Omega, \beta}^{A} f\right\|_{\dot{E}^{q, \lambda}} \leq C\|A\|_{C \dot{B} M O^{p_{2}, \lambda_{2}}}\|f\|_{\dot{E}^{p_{1}, \lambda_{1}}},
$$

where the constant $C$ is independent of $f$ and $A$. 
In order to prove Theorems 12 and 13, by a standard argument, we have the following lemma.

Lemma 14 (see [16]). Suppose $f \in C \dot{B} M O^{p, \lambda}, 1 \leq p<\infty$, $\lambda<1 / n$, and $r_{1}, r_{2} \in R^{+}$; then

$$
\begin{gathered}
\left(\frac{1}{\left|B\left(0, r_{1}\right)\right|^{1+p \lambda}} \int_{B\left(0, r_{1}\right)}\left|f(x)-f_{B\left(0, r_{2}\right)}\right|^{p} d x\right)^{1 / p} \\
\leq C\left(1+\left|\log \frac{r_{1}}{r_{2}}\right|\right)\|f\|_{C \dot{B} M O^{p, \lambda} .}
\end{gathered}
$$

Proof of Theorem 12. For any $R>0$, we denote $B(0, R)$ by $B$ and $B(0, k R)$ by $k B$ for any $k \in Z^{+}$. Thus we have the following estimates:

$$
\begin{aligned}
& \frac{1}{|B|} \int_{B}\left|\mathscr{H}_{\Omega, A, \beta}^{m} f(x)\right|^{q} d x \\
& \leq \frac{1}{|B|} \int_{B} \mid \frac{1}{|x|^{n-\beta}} \int_{B(0,|x|)} \frac{|\Omega(x-y) f(y)|}{|x-y|^{m-1}} \\
& \times\left.\left|R_{m}(A ; x, y)\right| d y\right|^{q} d x \\
& =\frac{1}{|B|} \sum_{k=-\infty}^{0} \int_{2^{k} B \backslash 2^{k-1} B} \mid \frac{1}{|x|^{n-\beta}} \sum_{i=-\infty}^{k} \int_{2^{i} B \backslash 2^{i-1} B} \frac{|\Omega(x-y) f(y)|}{|x-y|^{m-1}} \\
& \leq \frac{1}{|B|} \sum_{k=-\infty}^{0} \int_{2^{k} B \backslash 2^{k-1} B} \mid \frac{1}{|x|^{n-\beta}} \sum_{i=-\infty}^{k-3} \int_{2^{i} B \backslash 2^{i-1} B} \frac{|\Omega(x-y) f(y)|}{|x-y|^{m-1}} \\
& \quad+\frac{1}{|B|} \times\left|\frac{1}{m} \sum_{k=-\infty}^{0} \int_{2^{k} B \backslash 2^{k-1} B}\right| \frac{(A ; x, y)|d y|^{q} d x}{k-\beta} \sum_{i=k-2} \int_{2^{i} B \backslash 2^{i-1} B} \frac{|\Omega(x-y) f(y)|}{|x-y|^{m-1}} \\
& \quad \times\left.\left|R_{m}(A ; x, y)\right| d y\right|^{q} d x
\end{aligned}
$$

For the term $I$, let $\widetilde{A}(x)=A(x)$ $\sum_{|\gamma|=m-1}(1 / \gamma !) m_{2^{k} B}\left(D^{\gamma} A\right) x^{\gamma}$. Then it is easy to see
$R_{m}(A ; x, y)=R_{m}(\widetilde{A} ; x, y)$. As $p_{2}>n$, then by Lemmas 8 and 9 , we have

$$
\begin{aligned}
\left|R_{m}(\widetilde{A} ; x, y)\right| \leq & \left|R_{m-1}(\widetilde{A} ; x, y)\right| \\
& +\sum_{|\gamma|=m-1} \frac{1}{\gamma !}\left|D^{\gamma} \widetilde{A}(y)\right||x-y|^{m-1} \\
\leq & C|x-y|^{m-1} \sum_{|\gamma|=m-1}\left[|\widetilde{Q}|^{\lambda_{2}}\left\|D^{\gamma} A\right\|_{\mathrm{CB} M O^{p_{2}, \lambda_{2}}}\right. \\
& \left.+\left|D^{\gamma}(\widetilde{A})(y)\right|\right]
\end{aligned}
$$

where $\widetilde{Q}(x, y)$ is the cube centered at $x$ and having diameter $5 \sqrt{n}|x-y|$.

As $x \in 2^{k} B \backslash 2^{k-1} B$ and $y \in 2^{i} B \backslash 2^{i-1} B$ with $i \leq k-3$, we have $|x-y| \sim|x| \sim\left|2^{k} B\right|^{1 / n}$ and $|x-y| \geq C_{1}\left|2^{i} B\right|^{1 / n}$.

Thus by the Hölder inequality and the condition $1-1 / p_{1}-$ $1 / p_{2}-1 / r=1 / r^{\prime}-\beta / n-1 / q>0$, we have

$$
\begin{aligned}
& I \leq C|B|^{-1} \\
& \times \sum_{k=-\infty}^{0} \int_{2^{k} B \backslash 2^{k-1} B} \mid \frac{1}{|x|^{n-\beta}} \\
& \times \sum_{i=-\infty}^{k-3} \int_{2^{i} B \backslash 2^{i-1} B} \frac{|\Omega(x-y) f(y)|}{|x-y|^{m-1}} \\
& \times|x-y|^{m-1} \\
& \times \sum_{|\gamma|=m-1}\left(|\widetilde{Q}|^{\lambda_{2}}\left\|D^{\gamma} A\right\|_{\mathrm{CBMO}^{p_{2}, \lambda_{2}}}\right. \\
& \left.+\left|D^{\gamma} \widetilde{A}(y)\right|\right)\left.d y\right|^{q} d x \\
& \leq C|B|^{-1} \\
& \times \sum_{k=-\infty}^{0} \int_{2^{k} B \backslash 2^{k-1} B} \mid \frac{1}{|x|^{n-\beta}} \\
& \times \sum_{i=-\infty}^{k-3} \int_{2^{i} B \backslash 2^{i-1} B}|\Omega(x-y) f(y)| \\
& \times \sum_{|\gamma|=m-1}\left(|\widetilde{Q}|^{\lambda_{2}}\right. \\
& \times\left\|D^{\gamma} A\right\|_{\mathrm{CB}^{\prime} \mathrm{O}^{p_{2}, \lambda_{2}}} \\
& \left.+\left|D^{\gamma} \widetilde{A}(y)\right|\right)\left.d y\right|^{q} d x
\end{aligned}
$$




$$
\begin{aligned}
& \leq C|B|^{-1} \\
& \times \sum_{k=-\infty}^{0} \int_{2^{k} B \backslash 2^{k-1} B} \frac{1}{|x|^{(n-\beta) q}} \\
& \times \mid \sum_{i=-\infty}^{k-3}\left(\int_{2^{i} B \backslash 2^{i-1} B}|\Omega(x-y)|^{r} d y\right)^{1 / r} \\
& \times\left(\int _ { 2 ^ { i } B \backslash 2 ^ { i - 1 } B } \sum _ { | \gamma | = m - 1 } \left(|x-y|^{\lambda_{2} n}\right.\right. \\
& \times\left\|D^{\gamma} A\right\|_{\mathrm{CBMO}^{p_{2}, \lambda_{2}}} \\
& \left.\left.+\left|D^{\gamma} \widetilde{A}(y)\right|\right)^{p_{2}} d y\right)^{1 / p_{2}} \\
& \times\left(\int_{2^{i} B \backslash 2^{i-1} B}|f(y)|^{p_{1}} d y\right)^{1 / p_{1}} \\
& \times\left.\left|2^{i} B\right|^{1-1 / p_{1}-1 / p_{2}-1 / r}\right|^{q} d x \text {. }
\end{aligned}
$$

Note the following fact:

$$
\begin{gathered}
\left(\int _ { 2 ^ { i } B \backslash 2 ^ { i - 1 } B } \sum _ { | \gamma | = m - 1 } \left(|x-y|^{\lambda_{2} n}\left\|D^{\gamma} A\right\|_{\mathrm{CBMMO}^{p_{2}, \lambda_{2}}}\right.\right. \\
\left.\left.+\left|D^{\gamma} \widetilde{A}(y)\right|\right)^{p_{2}} d y\right)^{1 / p_{2}} \\
\leq C \sum_{|\gamma|=m-1}\left[\left\|D^{\gamma} A\right\|_{\mathrm{CBMOO}^{p_{2}, \lambda_{2}}\left|2^{k} B\right|^{\lambda_{2}}\left|2^{i} B\right|^{1 / p_{2}}}+\left(\int_{2^{i} B}\left|D^{\gamma} A(y)-m_{\widetilde{\mathrm{Q}}}\left(D^{\gamma} A\right)\right|^{p_{2}} d y\right)^{1 / p_{2}}\right] \\
\leq C \sum_{|\gamma|=m-1}\left\|D^{\gamma} A\right\|_{\mathrm{CB}^{2} \mathrm{O}^{p_{2}, \lambda_{2}}}\left|2^{i} B\right|^{1 / p_{2}}\left(\left|2^{k} B\right|^{\lambda_{2}}+\left|2^{i} B\right|^{\lambda_{2}}\right),
\end{gathered}
$$

where the last inequality follows from Lemma 14 and the fact $|x-y| \geq C_{1}\left|2^{i} B\right|^{1 / n}$. Thus by the condition $1+q \lambda>0$ and $\lambda_{1}+1>\lambda_{1}+1 / p_{1}>0$, we have

$$
\begin{aligned}
I \leq & C|B|^{-1} \\
& \times \sum_{k=-\infty}^{0}\left|2^{k} B\right|^{(\beta / n-1) q}
\end{aligned}
$$

$$
\begin{aligned}
& \times \int_{2^{k} B \backslash 2^{k-1} B}\left(\sum_{i=-\infty}^{k-3}\|f\|_{\dot{E}^{p_{1}}, \lambda_{1}}\left|2^{i} B\right|^{1 / p_{1}+\lambda_{1}}\left|2^{i} B\right|^{1 / r}\right. \\
& \times \sum_{|\gamma|=m-1}\left\|D^{\gamma} A\right\|_{\mathrm{CBMO}^{p_{2}, \lambda_{2}}}\left|2^{i} B\right|^{1 / p_{2}} \\
& \times\left(\left|2^{k} B\right|^{\lambda_{2}}+\left|2^{i} B\right|^{\lambda_{2}}\right) \\
& \left.\times\left|2^{i} B\right|^{1-1 / p_{1}-1 / p_{2}-1 / r}\right)^{q} d x \\
& \leq C\|f\|_{\dot{E}^{p_{1}, \lambda_{1}}}^{q} \sum_{|\gamma|=m-1}\left\|D^{\gamma} A\right\|_{\mathrm{CBMO}^{p_{2}, \lambda_{2}}}^{q}|B|^{-1} \\
& \times \sum_{k=-\infty}^{0}\left|2^{k} B\right|^{(\beta / n-1) q}\left|2^{k} B\right|\left|2^{k} B\right|^{\lambda_{2} q} \\
& \times\left(\sum_{i=-\infty}^{k-3}\left|2^{i} B\right|^{1 / p_{1}+\lambda_{1}}\right. \\
& \left.\times\left|2^{i} B\right|^{1 / p_{2}}\left|2^{i} B\right|^{1 / r}\left|2^{i} B\right|^{1-1 / p_{1}-1 / p_{2}-1 / r}\right)^{q} \\
& \leq C\|f\|_{\dot{E}^{p_{1}, \lambda_{1}}}^{q} \sum_{|\gamma|=m-1}\left\|D^{\gamma} A\right\|_{\mathrm{CBMO}^{p_{2}, \lambda_{2}}}^{q} \\
& \times|B|^{-1+\beta q / n-q+\lambda_{2} q+1+\lambda_{1} q+q} \\
& \times \sum_{k=-\infty}^{0} 2^{n k \beta q / n-k n q+k n+k \lambda_{2} n q+k \lambda_{1} n q+k n q} \\
& \leq C \sum_{|\gamma|=m-1}\left\|D^{\gamma} A\right\|_{\mathrm{CBMO}^{p_{2}, \lambda_{2}}}^{q}\|f\|_{\dot{E}^{p_{1}, \lambda_{1}}}^{q}|B|^{q \lambda} .
\end{aligned}
$$

To estimate the term $I I$, we adopt some basic ideas from the estimates of the term $I_{2}$ in Theorem 3. First, we denote $R_{m}(A ; x, y)=R_{m}\left(A_{k}^{\phi} ; x, y\right)=R_{m}\left(A_{k} ; x, y\right)$ for $x \in 2^{k} B$ and $y \in 2^{i} B$ with $k-2 \leq i \leq k$, where $A_{k}^{\phi}(x)=$ $R_{m-1}\left(A_{k} ; x, y_{0}\right) \phi\left(\left|x-y_{0}\right|^{-1} x\right)$ with $y_{0} \in 2^{i} B \backslash 2^{i-1} B$. Here $A_{k}(x)=A(x)-\sum_{|\gamma|=m-1}(1 / \gamma !) m_{2^{k} B}\left(D^{\gamma} A\right) x^{\gamma}$ and $\phi$ is defined as in Section 2.

As $\Omega \in L^{r}\left(\mathbb{S}^{n-1}\right)$ with $r>p_{1}^{\prime}$, by Lemma 7 , we get

$$
\begin{aligned}
I I & \leq \frac{1}{|B|} \sum_{k=-\infty}^{0} \sum_{i=k-2}^{k}\left\|\mathscr{H}_{\Omega, A_{k}^{\phi}, \beta}^{m} f_{i}\right\|_{q}^{q} \\
& \leq \frac{C}{|B|} \sum_{k=-\infty}^{0} \sum_{i=k-2}^{k}\left\|f_{i}\right\|_{L^{p_{1}}}^{q} \sum_{|\gamma|=m-1}\left\|D^{\gamma} A_{k}^{\phi}\right\|_{L^{p_{2}}}^{q},
\end{aligned}
$$

where $f_{i}$ is defined by $f_{i}(x)=f(x) \chi_{2^{i} B \backslash 2^{i-1} B}(x)$. 
For $D^{\gamma} A_{k}^{\phi}$, as $\left|x-y_{0}\right| \leq C\left|y_{0}\right|$, then by Lemmas 8 and 14 , we have the following estimates:

$$
\begin{aligned}
& \quad\left|D^{\gamma} A_{k}^{\phi}(x)\right| \\
& \leq \sum_{|\mu|+|\nu|=m-1} C_{\mu \nu}\left|R_{m-1-|\mu|}\left(D^{\mu} A_{k} ; x, y_{0}\right)\right| \\
& \quad \times\left|D^{\nu} \phi\left(\left|x-y_{0}\right|^{-1} x\right)\right|\left|x-y_{0}\right|^{-|\nu|} \\
& \leq \sum_{|\mu|+|\nu|=m-1}\left|x-y_{0}\right|^{m-1-|\mu|-|\nu|} \\
& \quad \sum_{\left|\gamma^{\prime}\right|=m-1-|\mu|}\left(\frac{1}{\left|\widetilde{Q}\left(x, y_{0}\right)\right|} \int_{\widetilde{Q}\left(x, y_{0}\right)}\left|D^{\gamma^{\prime}}\left(D^{\mu} A_{k}\right)(z)\right|^{p_{2}} d z\right)^{1 / p_{2}} \\
& \quad \times \chi_{|x| \leq\left|x-y_{0}\right|}(x) \\
& \leq \sum_{|\gamma|=m-1}\left|D^{\gamma} A \|_{C \dot{M} O^{p_{2}, \lambda_{2}}}\right| x-\left.y_{0}\right|^{\lambda_{2} n} \chi_{|x| \leq C\left|y_{0}\right|}(x) .
\end{aligned}
$$

Thus we have

$$
\begin{aligned}
& \sum_{|\gamma|=m-1}\left\|D^{\gamma} A_{k}^{\phi}\right\|_{L^{p_{2}}}^{q} \\
& \quad \leq C \sum_{|\gamma|=m-1}\left\|D^{\gamma} A\right\|_{\mathrm{CBMO}^{p_{2}, \lambda_{2}}}^{q}\left|2^{i} B\right|^{q \lambda_{2}}\left|2^{i} B\right|^{q / p_{2}} .
\end{aligned}
$$

By the above estimates, we obtain

$$
\begin{aligned}
I I \leq & \frac{C}{|B|} \sum_{k=-\infty}^{0} \sum_{i=k-2}^{k}\left(\frac{1}{\left|2^{i} B\right|^{1+p_{1} \lambda_{1}}} \int_{2^{i} B}|f(x)|^{p_{1}} d x\right)^{q / p_{1}} \\
& \times\left|2^{i} B\right|^{q / p_{1}+q \lambda_{1}} \\
& \times \sum_{|\gamma|=m-1}\left\|D^{\gamma} A\right\|_{\mathrm{CBMO}^{p_{2}, \lambda_{2}}}^{q}\left|2^{i} B\right|^{q \lambda_{2}+q / p_{2}} \\
\leq & C \sum_{k=-\infty}^{0} \sum_{i=k-2}^{k} 2^{i n(1+\lambda q)} \\
& \times \sum_{|\gamma|=m-1}\left\|D^{\gamma} A\right\|_{\mathrm{CBMMO}^{p_{2}, \lambda_{2}}}^{q}\|f\|_{\dot{E}^{p_{1}, \lambda_{1}}}^{q}|B|^{q \lambda} \\
\leq & C \sum_{|\gamma|=m-1}\left\|D^{\gamma} A\right\|_{\mathrm{CBMO}^{p_{2}, \lambda_{2}}}^{q}\|f\|_{\dot{E}^{p_{1}, \lambda_{1}}}^{q}|B|^{q \lambda} .
\end{aligned}
$$

Combining the estimates of $I$ and $I I$ and by the definition of $\dot{E}^{q, \lambda}$, we finish the proof of Theorem 12 .

Proof of Theorem 13. The proof of Theorem 13 is quite similar but much simpler than Theorem 12 and we omit the details here.

\section{Acknowledgments}

This work was supported by the National Natural Science Foundation of China under Grant nos. 10931001, 11226104, and 11271175, the Natural Science Foundation of Jiangxi Province under Grant no. 20114BAB211007, and the Science Foundation of Jiangxi Education Department under Grant no. GJJ13703. This work was also supported by the Key Laboratory of Mathematics and Complex System (Beijing Normal University), Ministry of Education, China.

\section{References}

[1] A. P. Calderón and A. Zygmund, "On singular integrals," American Journal of Mathematics, vol. 78, pp. 289-309, 1956.

[2] R. R. Coifman, R. Rochberg, and G. Weiss, "Factorization theorems for Hardy spaces in several variables," Annals of Mathematics, vol. 103, no. 3, pp. 611-635, 1976.

[3] J. Cohen, "A sharp estimate for a multilinear singular integral in $\mathbb{R}^{n}$, , Indiana University Mathematics Journal, vol. 30, no. 5, pp. 693-702, 1981.

[4] J. Cohen and J. Gosselin, "A BMO estimate for multilinear singular integrals," Illinois Journal of Mathematics, vol. 30, no. 3, pp. 445-464, 1986.

[5] S. Lu and Q. Wu, "CBMO estimates for commutators and multilinear singular integrals," Mathematische Nachrichten, vol. 276, pp. 75-88, 2004.

[6] D. Y. Yan, Some problems on multilinear singular integral operators and multilinear oscillatory singular integral operators [Ph.D. thesis], Beijing Normal University, Beijing, China, 2001.

[7] X. Yu and X. X. Tao, "Boundedness for a class of generalized commutators on $\lambda$-central Morrey space," Acta Mathematica Sinica. English Series, vol. 29, no. 10, pp. 1917-1926, 2013.

[8] C. Wang and Z. Zhang, "A new proof of Wu's theorem on vortex sheets," Science China, vol. 55, no. 7, pp. 1449-1462, 2012.

[9] S. Wu, "Mathematical analysis of vortex sheets," Communications on Pure and Applied Mathematics, vol. 59, no. 8, pp. 10651206, 2006.

[10] G. H. Hardy, "Note on a theorem of Hilbert," Mathematische Zeitschrift, vol. 6, no. 3-4, pp. 314-317, 1920.

[11] Z.-W. Fu, Z.-G. Liu, S.-Z. Lu, and H.-B. Wang, "Characterization for commutators of $n$-dimensional fractional Hardy operators," Science in China A, vol. 50, no. 10, pp. 1418-1426, 2007.

[12] M. Christ and L. Grafakos, "Best constants for two nonconvolution inequalities," Proceedings of the American Mathematical Society, vol. 123, no. 6, pp. 1687-1693, 1995.

[13] S. Lu and D. Yang, "The central BMO spaces and LittlewoodPaley operators," Approximation Theory and its Applications, vol. 11, no. 3, pp. 72-94, 1995.

[14] Y. Komori, "Notes on singular integrals on some inhomogeneous Herz spaces," Taiwanese Journal of Mathematics, vol. 8, no. 3, pp. 547-556, 2004.

[15] S. Z. Lu and F. Y. Zhao, "CBMO estimates for multilinear Hardy operators," Acta Mathematica Sinica. English Series, vol. 26, no. 7, pp. 1245-1254, 2010.

[16] G. L. Gao and X. Yu, "Some estimates for the generalized Hardy operators on some function spaces," Acta Mathematica Sinica. Chinese Series, vol. 55, no. 6, pp. 1101-1110, 2012.

[17] X. Yu and S. Lu, "Endpoint estimates for generalized commutators of Hardy operators on $H^{1}$ space," Journal of Function Spaces and Applications, vol. 2013, Article ID 410305, 11 pages, 2013. 
[18] S. Z. Lu and D. C. Yang, "The decomposition of weighted Herz space and its applications," Science in China A, vol. 38, no. 2, pp. 147-158, 1995.

[19] Z. Fu, S. Lu, and F. Zhao, "Commutators of $n$-dimensional rough Hardy operators," Science China, vol. 54, no. 1, pp. 95-104, 2011.

[20] Y. Ding, "A note on multilinear fractional integrals with rough kernel," Advances in Mathematics, vol. 30, no. 3, pp. 238-246, 2001.

[21] N. Wiener, "Generalized harmonic analysis," Acta Mathematica, vol. 55, no. 1, pp. 117-258, 1930.

[22] A. Beurling, "Construction and analysis of some convolution algebras," Annales de l'Institut Fourier, vol. 14, pp. 1-32, 1964.

[23] H. Feichtinger, "An elementary approach to Wiener's third Tauberian theorem on Euclidean n-space," in Proceedings, Conference at Cortona 1984, vol. 29 of Symposia Mathematica, pp. 267-301, Academic Press, New York, NY, USA, 1987.

[24] Y. Z. Chen and K.-S. Lau, "Some new classes of Hardy spaces," Journal of Functional Analysis, vol. 84, no. 2, pp. 255-278, 1989.

[25] J. García-Cuerva, "Hardy spaces and Beurling algebras," Journal of the London Mathematical Society, vol. 39, no. 3, pp. 499-513, 1989.

[26] J. Alvarez, J. Lakey, and M. Guzmán-Partida, "Spaces of bounded $\lambda$-central mean oscillation, Morrey spaces, and $\lambda$ central Carleson measures," Collectanea Mathematica, vol. 51, no. 1, pp. 1-47, 2000.

[27] Z. W. Fu, Y. Lin, and S. Z. Lu, " $\lambda$-central BMO estimates for commutators of singular integral operators with rough kernels," Acta Mathematica Sinica. English Series, vol. 24, no. 3, pp. 373386, 2008.

[28] Z. W. Fu, S. Z. Lu, and W. Yuan, "A weighted variant of Riemann-Liouville fractional integrals on $R^{n}$, , Abstract and Applied Analysis, vol. 2012, Article ID 780132, 18 pages, 2012. 


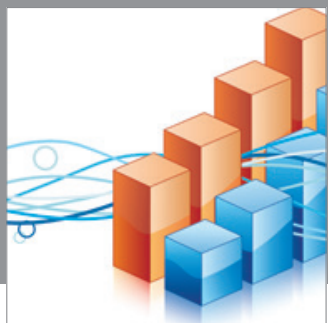

Advances in

Operations Research

mansans

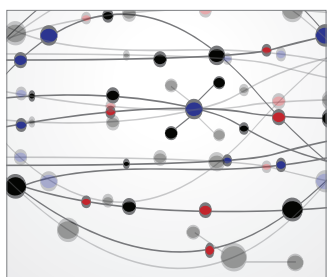

The Scientific World Journal
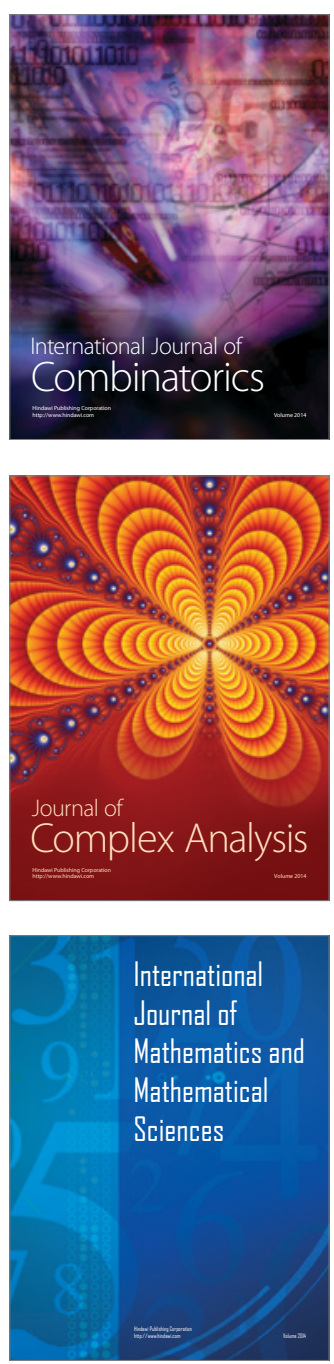
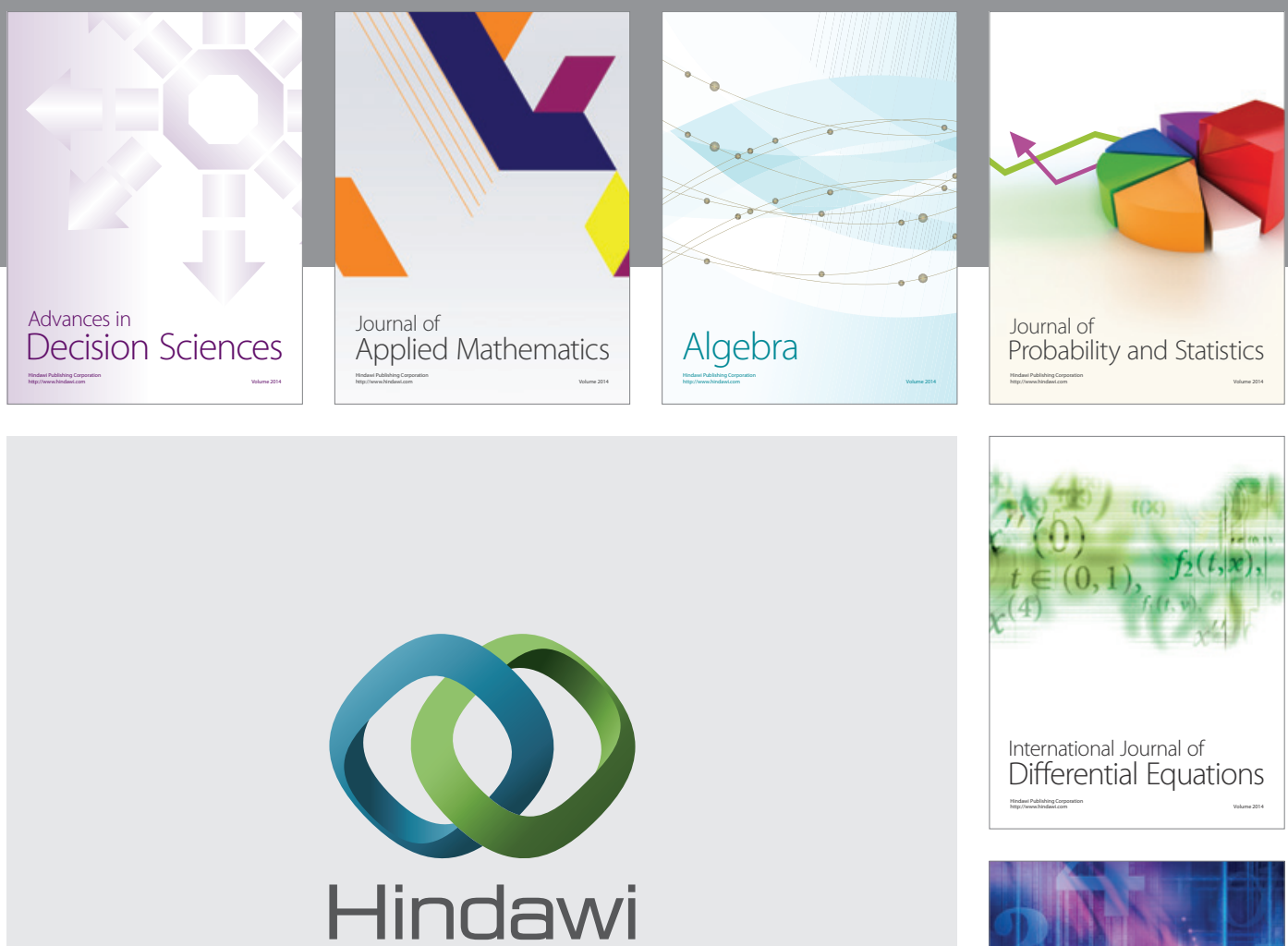

Submit your manuscripts at http://www.hindawi.com
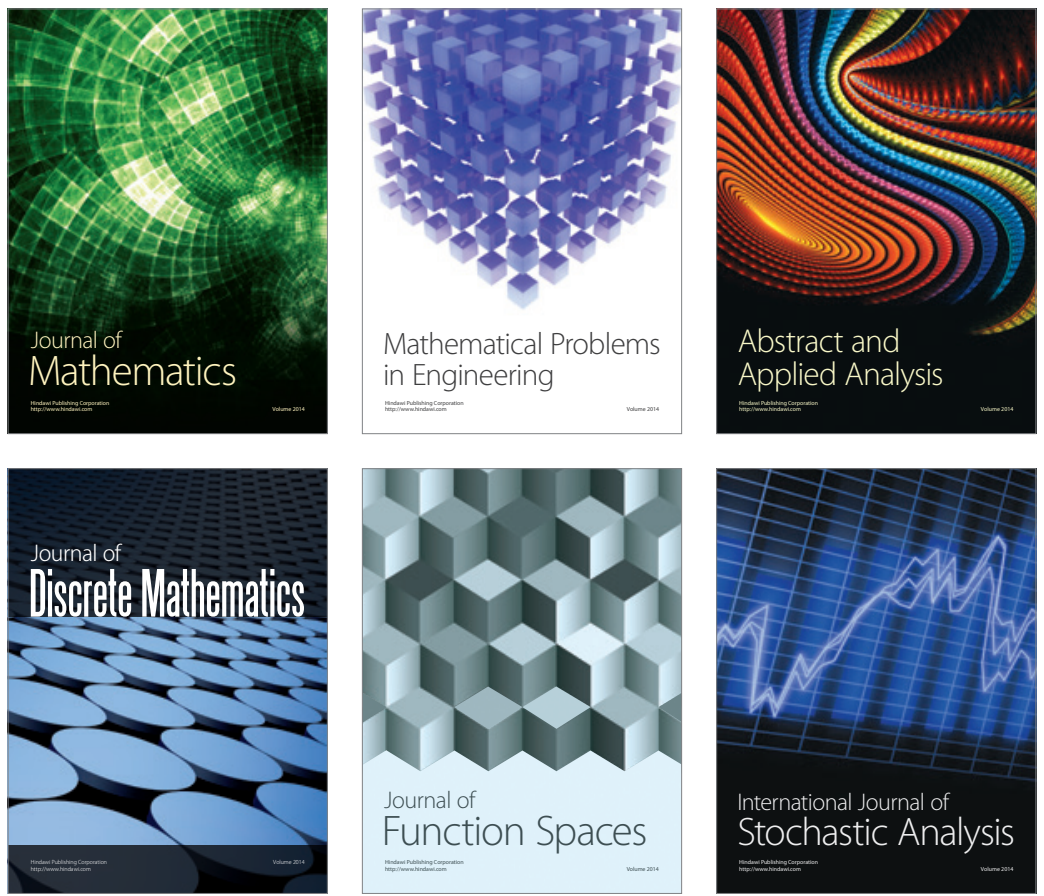

Journal of

Function Spaces

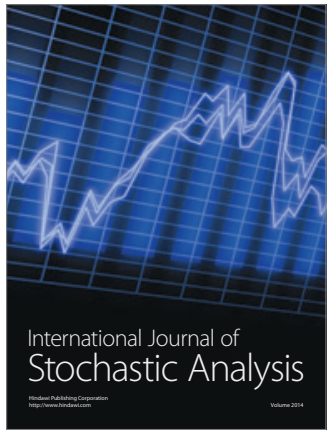

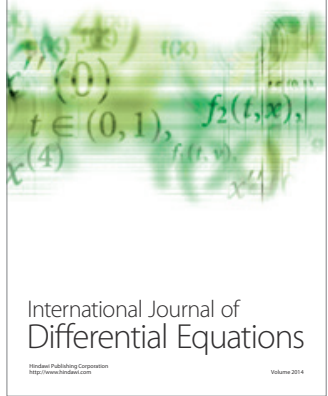
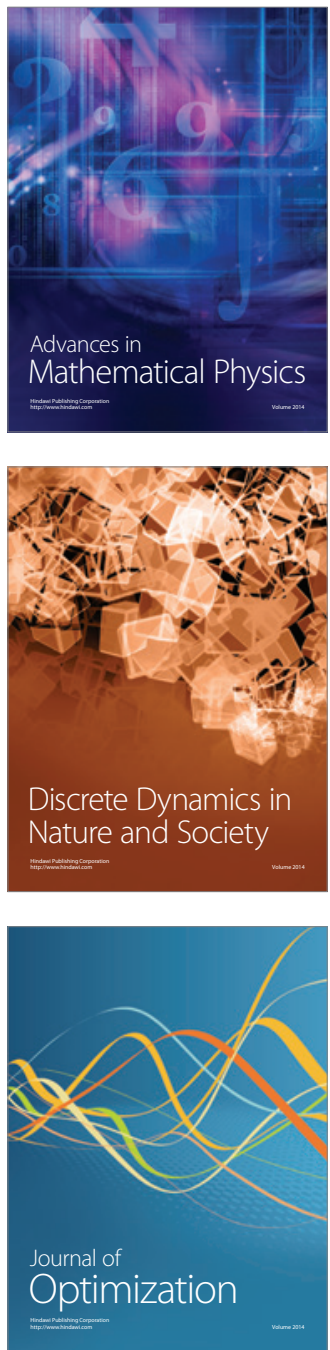